\title{
Cryptococcal Infection with Ruxolitinib in Primary Myelofibrosis: A Case Report and Literature Review
}

\author{
Zachary Ciochetto $^{1}$, Njeri Wainaina ${ }^{1}$, Anna Corey ${ }^{1}$, Mary Beth Graham ${ }^{1}$, and Muhammad \\ Bilal Abid ${ }^{1}$ \\ ${ }^{1}$ Medical College of Wisconsin
}

December 9, 2021

\begin{abstract}
Cryptococcus neoformans $(\mathrm{CN})$ is an encapsulated yeast that causes disseminated and potentially life-threatening in immunocompromised hosts. We present a patient with primary myelofibrosis on ruxolitinib who developed disseminated disease due to CN. The report underscores the importance of suspecting infections with intracellular pathogens in immunosuppressed patients on ruxolitinib.
\end{abstract}

Cryptococcal Infection with Ruxolitinib in Primary Myelofibrosis: A Case Report and Literature Review Zachary Ciochetto $^{1}$, Njeri Wainaina ${ }^{1}$, Mary Beth Graham ${ }^{1}$, Anna Corey ${ }^{1}$, and Muhammad Bilal Abid ${ }^{1}$

${ }^{1}$ Division of Infectious Diseases, Medical College of Wisconsin, Milwaukee, WI, USA

Corresponding author:

Zachary Ciochetto, MD

Division of Infectious Diseases. Medical College of Wisconsin. 8701 W Watertown Plank Rd, Milwaukee, WI, 53226

Telephone: 586-321-5949. Fax: 414-955-0093

Email: zciochetto@mcw.edu

Alternate corresponding author:

Muhammad Bilal Abid, MD

Division of Infectious Diseases. Medical College of Wisconsin. 8701 W Watertown Plank Rd, Milwaukee, WI, 53226

Telephone: 586-321-5949. Fax: 414-955-0093

Email: mabid@mcw.edu

Article Category: Case Report and Literature review

Abstract word count: 110

Manuscript word count: 1155

References: 16

Tables: 1 
Figures: 0

\section{ABSTRACT:}

Cryptococcus neoformans $(\mathrm{CN})$ is an encapsulated yeast that is found worldwide. It causes self-limiting infections in immunocompetent hosts, however, infections due to CN could be disseminated and potentially life-threatening in immunocompromised hosts. Herein, we present a patient with primary myelofibrosis who received ruxolitinib and developed disseminated cryptococcosis due to CN. We further discuss immune compromising factors indigenous to myeloproliferative neoplasms, ruxolitinib, and immunological pathways associated with janus kinase inhibition. We further review other cases of cryptococcal infections in patients receiving ruxolitinib reported in the literature. The report underscores the importance of suspecting infections with intracellular pathogens early in the course of illness in patients with higher rates of cumulative immunosuppression.

\section{INTRODUCTION:}

The Cryptococcus species complex are encapsulated yeasts comprising two pathogenic species, Cryptococcus neoformans $(\mathrm{CN})$ and Cryptococcus gattii $(\mathrm{CG})$. $\mathrm{CN}$ is distributed globally in soil, particularly when enriched with bird droppings, while CG is endemic to tropical and sub-tropical climates. Cryptococcosis is acquired via inhalation of the spores from the soil with inoculation of the host's lungs leading to an asymptomatic or mild self-limiting infection if immunocompetent. In contrast to immunocompromised individuals, it can lead to severe primary or reactivated disease. The highest morbidity and mortality are seen with dissemination to the central nervous system causing meningitis, but it can also involve the skin, bone, spleen, and other tissues [1].

Cryptococcosis is an acquired immunodeficiency syndrome (AIDS) defining infection and is also associated with conditions that disrupt effector T-cell function such as solid organ and hematopoietic cell transplantation, progressive cancers, and prolonged corticosteroid use. Immune defense mechanisms against cryptococcus fall within innate and adaptive pathway, both of which the fungus attempts to evade. The fungus attempts to evade the immune system using several mechanisms, one of which is the polysaccharide capsule [2]. Th1 and Th2 cells, along with cytokines, directly activate CD8+ and natural killer (NK) cells which perform the fungicidal activity through perforins and granzymes [2]. Hosts with insufficient quantity or quality of T-cells develop disseminated infection as they are unable to mount this response.

Myeloproliferative neoplasms (MPNs) are stem cell-derived clonal myeloid malignancies characterized by a unique somatic mutational profile with three usually mutually exclusive mutations (JAK2, MPL, and CALR). These driver mutations result in constitutive activation of Janus kinase (JAK2)-dependent signaling.

Primary myelofibrosis (PMF) is a variant of MPNs that develops as the bone marrow is replaced by fibrotic material resulting in extramedullary hematopoiesis. Ruxolitinib is a selective oral inhibitor of both JAK1/2 protein kinases. Its use in patients with PMF is associated with a reduction in spleen volume as well as improvement in symptoms and survival. Evidence shows that ruxolitinib exerts potent anti-inflammatory and immunosuppressive effects. Cellular targets of ruxolitinib include components of both the innate and adaptive immune system, such as NK and DCs, helper T-cells, and regulatory T-cells (Tregs) [3].

Herein, we present a patient who developed disseminated cryptococcosis while receiving ruxolitinib therapy for PMF. We further present relevant literature and discuss immunologic mechanisms involved in the fungal pathogenesis in immunocompromised patients.

\section{CASE PRESENTATION:}

An 82-year-old man with a past medical history of PMF was admitted to the hospital with headache, nausea, and vomiting for 5 days. Five years prior, he had been diagnosed with high-risk PMF with severe reticulin fibrosis on bone marrow morphology and positive JAK2 and CALR mutations. He received hydroxyurea initially for one year and then received low dose corticosteroids and ruxolitinib, due to worsening anemia, thrombocytopenia, leukocytosis, and splenomegaly. In the emergency department, he was afebrile, blood 
pressure was 176/106 $\mathrm{mmHg}$, and pulse was 51 beats per minute, with normal oxygenation and respiratory rate. The patient did not exhibit cachexia, lymphadenopathy, jaundice, icterus, or hepatomegaly. Physical exam was unremarkable other than mild non-tender splenomegaly.

Initial laboratory testing was notable for sodium of $125 \mathrm{mEq} / \mathrm{L}$ (normal 135-145 mEq/L), serum osmolality $260 \mathrm{mOsm} / \mathrm{kg}$ (normal 285-295 mOsm $/ \mathrm{kg}$ ), lactate dehydrogenase (LDH) 974 unit/L (normal 135-225 unit/L), hemoglobin $7.7 \mathrm{~g} / \mathrm{dL}$ (normal male 13.5-17.5 g/dL) and thrombocytopenia at $69 \mathrm{x} 10^{9} / \mathrm{L}$ (normal $150-450 \times 10^{9} / \mathrm{L}$ ). White blood cell (WBC) count was $32.9 \times 10^{9} / \mathrm{L}$ (normal $4-12 \times 10^{9} / \mathrm{L}$ ) with absolute neutrophilia at $15.79 \times 10^{9} / \mathrm{L}$, and lymphocytosis at $1.32 \times 10^{9} / \mathrm{L}$. Head and contrasted visceral imaging were unremarkable and blood cultures were obtained.

The patient developed seizures 5 days into the treatment for syndrome of inappropriate antidiuretic hormone, prompting further investigation. Computerized tomography (CT) and magnetic resonance imaging (MRI) of the patients' brain were negative along with unremarkable electroencephalography (EEG). CT chest revealed bilateral pulmonary nodules. Blood cultures grew yeast two days after acquisition, so intravenous (IV) micafungin $100 \mathrm{mg}$ daily was initiated, and a serum cryptococcal antigen was tested. On the $7^{\text {th }}$ day of hospitalization, the yeast in blood cultures was identified as $\mathrm{CN}$ and the serum cryptococcal antigen returned positive with titers of 1:>1024 (normal $<1: 2$ ). In consultation with infectious diseases, the antimicrobial regimen was adjusted to IV liposomal amphotericin-B $3 \mathrm{mg} / \mathrm{kg}$ daily and flucytosine $25 \mathrm{mg} / \mathrm{kg}$ twice daily with recommendations for a lumbar puncture. Unfortunately, the patient succumbed to overwhelming sepsis prior to undergoing lumbar puncture or receiving the suggested antifungal agents.

\section{DISCUSSION:}

We present a patient who developed irreversible sepsis from suspected disseminated cryptococcal infection due to CN temporally associated with the use of ruxolitinib for PMF. Cell-mediated immunity is essential in preventing severe infection with intracellular pathogens such as CN. The case underpins the immunological principle of compounding immunosuppression.

In PMF with dysregulated JAK1/2 pathways, myeloid cells show altered expression of both chemokine and cytokine receptors along with reduced ability to produce inflammatory cytokines in response to infectious stimuli. This phenomenon reduces cell proliferation and migration to infected tissues with reduced activation of NK cells, impairing the hosts defenses. This can be compounded by ruxolitinib inhibition of IL- 4 which negatively effects downstream signaling and differentiation of monocytes into NK cells. This compounding immunosuppression thus increases the hosts risk for infections [4-5].

Treatment of PMF with ruxolitinib acts by inhibiting the JAK1/2 pathways, which prevents abnormal hematopoiesis [6-7]. Concurrent, modulation of critical JAK pathways and downstream signaling compromises both innate and adaptive immune systems, predisposing patients to bacterial, viral, and fungal infections which are the main cause of morbidity and mortality in patients with PMF [8]. The higher the International Prognostic Scoring System (IPSS) and greater the spleen volume, the higher the risk of infection [9-10]. Pivotal clinical trials (COMFORT-I, COMFORT-II, and JUMP) showed that patients with PMF treated with ruxolitinib had reduction in spleen size, decrease in constitutional symptoms, and improved survival [14-16].

The COMFORT-I and COMFORT-II trials also reported an increased risk of bacterial and viral infections in patients treated with ruxolitinib [14-15]. COMFORT-II reported higher incidence of herpes zoster infections (11.5\% of patients), pneumonia (13.1\%), sepsis (7.6\%), and urinary tract infections $(24.6 \%)$, with two cases of tuberculosis. It was recognized that myeloperoxidase deficiency was seen with homozygous CALR mutations, leading to neutrophil dysfunction. With diminished functional efficacy of neutrophils, DCs, NK cells, and Th1 and Th17 cells, patients are at a higher risk for disseminated viral, and fungal infections.

Additionally, we performed a literature review of invasive cryptococcal infections associated with immunosuppressive agents such as ruxolitinib. There are two published reports of cryptococcal pulmonary infection and three of cryptococcal meningitis (Table 1 ) [11-15]. Our patient, along with other cases in table 1 
, highlights the need for increased surveillance for intracellular pathogens, continued antimicrobial prophylaxis, and high clinical suspicion for fungal infections in patients with PMF receiving ruxolitinib. Providers caring for patients with MPNs receiving ruxolitinib need to pursue a personalized risk-adapted approach in gauging ruxolitinib's morbidity and mortality benefits against the risks for opportunistic infections. Further data on epidemiology and severity of infections seen with ruxolitinib use in patients with MPNs is needed.

\section{Abbreviations:}

CN: Cryptococcus neoformans

CG: Cryptococcus gattii

PMF: Primary myelofibrosis

MPN: Myeloproliferative neoplasm

JAK: Janus kinase

MPL: thrombopoietin receptor gene

CALR: Calreticulin

NK: Natural killer

IFI: Invasive fungal infection

HIV: human-immunodeficiency virus

FDA: US Food and Drug Administration

Tregs: T-regulatory cells

ID: Infectious diseases

IPSS: International Prognostic Scoring System

\section{Declarations:}

Ethics approval and consent to participate: Not applicable.

Consent for publication: Written informed consent was obtained from the patient for publication of this case report and any accompanying images. A copy of the written consent is available for review by the Editor of this journal.

Availability of data and material: Data sharing is not applicable to this article as no datasets were generated or analyzed during the current study.

Competing interests: All authors report no conflicts of interest relevant to this article.

Funding: None.

Authors' contributions: ZC put together the manuscript with the help of MBA. Manuscript was reviewed and edited with the help of NW and MBG.

Acknowledgements: Not applicable.

REFERENCES:

1. Park, B. J., K. A. Wannemuehler, B. J. Marston, N. Govender, P. G. Pappas, T. M. Chiller, et al. 2009. Estimation of the current global burden of cryptococcal meningitis among persons living with HIV/ AIDS. AIDS 23:525-530. 
2. Voelz, K., and May, R.C. 2010. Cryptococcal interactions with the host immune system. Eukaryot. Cell 9:835-846.

3. Wang, J.C., Sindhu, H., Chen, C., Kundra, A., Kafeel, M., Wong, C., et al. Immune derangements in patients with myelofibrosis: The role of Treg, Th17, and sIL2R $\alpha$. PLoS One . 2015;10(3).

4. Barone, M., Catani, L., Ricci, F., Romano, M., Forte, D., Auteri, G., et al. The role of circulating monocytes and JAK inhibition in the infectious-driven inflammatory response of myelofibrosis. Oncoimmunology . 2020;9(1).

5. Lussana, F., Cattaneo, M., Rambaldi, A., Squizzato, A. Ruxolitinib-associated infections: A systematic review and meta-analysis. Am J Hematol . 2018;93(3):339-347.

6. Harrison, C.N., Vannucchi, A.M., Kiladjian, J.J., Al-Ali, H.K., Gisslinger, H., Knoops, L., et al. Long-term findings from COMFORT-II, a phase 3 study of ruxolitinib vs best available therapy for myelofibrosis. Leukemia . 2016;30(8):1701-1707.

7. Al-Ali, H.K., Griesshammer, M., le Coutre, P., Waller, C.F., Liberati, A.M., Schafhausen, P., et al. Safety and efficacy of ruxolitinib in an open-label, multicenter, single-arm phase $3 \mathrm{~b}$ expanded-access study in patients with myelofibrosis: A snapshot of 1144 patients in the JUMP trial. Haematologica . 2016;101(9):1065-1073.

8. Abid, M.B., De Mel, S., Limei, M.P. Disseminated Cryptococcal infection in an immunocompetent host mimicking plasma cell disorder: a case report and literature review. Clin Case Reports . 2015;3(5):319324.

9. Abid, M.B., Stromich, J., Gundacker, N.D. Is ibrutinib associated with disseminated cryptococcosis with CNS involvement? Cancer Biol Ther . 2019;20(2):138-140.

10. Polverelli, N., Breccia, M., Benevolo, G., Martino, B., Tieghi, A., Latagliata, R., et al. Risk factors for infections in myelofibrosis: role of disease status and treatment. A multicenter study of 507 patients. Am J Hematol . 2017;92(1):37-41.

11. Wysham, N.G., Sullivan, D.R., Allada, G. An opportunistic infection associated with ruxolitinib, a novel janus kinase 1,2 inhibitor. Chest. 2013;143 (5):1478-1479.

12. Chen, C.C., Chen, Y.Y., Huang, C.E. Cryptococcal meningoencephalitis associated with the long-term use of ruxolitinib. Ann Hematol. 2016;95 (2):361-362.

13. Hirano, A., Yamasaki, M., Saito, N., Iwato, K., Daido, W., Funaishi, K., et al. Pulmonary cryptococcosis in a ruxolitinib-treated patient with primary myelofibrosis. Respir Med Case Rep. 2017;22 :87-90.

14. Tsukui, D., Fujita, H., Suzuki, K., Hirata, K. A case report of cryptococcal meningitis associated with ruxolitinib. Medicine (Baltimore) . 2020;99(13):e19587.

15. Prakash, K., Richman, D. A case report of disseminated histoplasmosis and concurrent cryptococcal meningitis in a patient treated with ruxolitinib. BMC Infect Dis . 2019;19(1):287.

16. Vannucchi, A.M., Kantarjian, H.M., Kiladjian, J.J., Gotlib, J., Cervantes, F., Mesa, R.A., et al. A pooled analysis of overall survival in COMFORT-I and COMFORT-II, 2 randomized phase III trials of ruxolitinib for the treatment of myelofibrosis. Haematologica . 2015;100(9):1139-1145.

\section{Hosted file}

Cryptococcus Patient Table.docx available at https://authorea.com/users/450442/articles/ 548739-cryptococcal-infection-with-ruxolitinib-in-primary-myelofibrosis-a-case-reportand-literature-review 